\title{
People of Opposing Views can Share Common Interests
}

\author{
Eduardo Graells-Garrido \\ Web Research Group \\ Universitat Pompeu Fabra \\ Barcelona, Spain \\ eduard.graells@upf .edu
}

\author{
Mounia Lalmas \\ Yahoo Labs \\ London, UK \\ mounia@acm.org
}

\author{
Daniele Quercia \\ Yahoo Labs \\ Barcelona, Spain \\ dquercia@yahoo-inc.com
}

\begin{abstract}
In online social networks, people tend to connect with likeminded people and read agreeable information. Direct recommendation of challenging content has not worked well because users do not value diversity and avoid challenging content. In this poster, we investigate the possibility of an indirect approach by introducing intermediary topics, which are topics that are common to people having opposing views on sensitive issues, i.e., those issues that tend to divide people. Through a case study about a sensitive issue discussed in Twitter, we show that such intermediary topics exist, opening a path for future work in recommendation promoting diversity of content to be shared.
\end{abstract}

\section{Categories and Subject Descriptors}

H.3.4 [Information Storage and Retrieval]: Systems and Software-Information networks

\section{Keywords}

Social Networks; Topic Modeling; Homophily; Intermediary Topics.

\section{INTRODUCTION}

Social sciences research has shown that, in social networks, people tend to connect with people with similar beliefs, a phenomena known as homophily, and prefer to read only agreeable information, a phenomena known as selective exposure. Both phenomena have been studied in online settings $[4,3,2]$. For instance, on the abortion issue, \#prolife users hardly interact with \#prochoice users in a debate context, although those users could engage in conversation about other interests, such as \#musicmonday.

Motivated by this scenario, we set out to suggest new connections among users with challenging points of view on sensitive issues, i.e., those issues which tend to divide people. We introduce the concept of intermediary topics, which are non-sensitive topics that might help to connect

Copyright is held by the International World Wide Web Conference Committee (IW3C2). IW3C2 reserves the right to provide a hyperlink to the author's site if the Material is used in electronic media. WWW'14 Companion, April 7-11, 2014, Seoul, Korea. ACM 978-1-4503-2745-9/14/04.

http://dx.doi.org/10.1145/2567948.2577368. people who have opposing views on sensitives issues but have similar views on intermediary topics. Previous work has focused on a direct approach to recommendation by displaying diverse and challenging information to users, but users do not value diversity [3] and still prefer agreeable and like-minded information [2]. Hence, an indirect approach through intermediary topics could be more feasible.

In this paper, we hypothesize that these intermediary topics exist and are measurable in the microblogging platform Twitter. We demonstrate that using topic modeling on user generated content and measures of centrality and diversity, it is possible to find and quantify these intermediary topics.

\section{METHODOLOGY}

A stance is defined as a position adopted with respect to something. An issue is said to be sensitive when stances about the issue tend to divide people. For instance, abortion is a sensitive issue in many countries, whereas musical taste is usually not. For a given sensitive issue, we collect relevant tweets based on keywords and hashtags that are associated with it. For instance, \#prolife and \#prochoice represent two abortion stances.

The collected tweets are used to construct several issue stance documents (i.e., concatenation of tweets from an issue stance) and user documents (i.e., concatenation of tweets authored by an user), which we represent as vectors. For each stance, we define a stance vector $\vec{s}$ in which each element refers to the importance of a given word $w$. For each user, we define a user vector $\vec{u}$, where each element refers to the importance of a given word $w$. In both definitions, word importance is weighted using TF-IDF with respect to the corpus of issue stances, as stances in different sensitive issues might be related because of ideology. We define the user stance $\overrightarrow{v_{i}}$ as a vector where each element $v_{j}$ corresponds to the cosine similarity between the user vector $\overrightarrow{u_{i}}$ and the stance vector $\overrightarrow{s_{j}}$.

Topic Graph. We explore the topical diversity of user documents by performing Latent Dirichlet Allocation [1] to its corpus. LDA is a generative model that, given a number of topics $k$ and a corpus, estimates which words contribute to each topic and which topics contribute to each document. We build an undirected topic graph where each LDA topic is a node, two nodes are connected if the two corresponding topics contribute to the same document, and edges are weighted based on the fraction of documents that contributed to it. We filter the edges based on their weight, leaving only those in the upper $10 \%$, and compute the betweenness centrality of nodes in the resulting graph. We define topic diversity 
with respect to a sensitive issue as the Shannon entropy $H=$ $-\sum_{i=1}^{N} p_{i} \log p_{i}$, where $p_{i}$ is the fraction of users in stance $i$ for a given issue, and $N$ is the number of issue stances for that particular sensitive issue. We define intermediary topics as topics whose betweenness centrality and topic diversity are higher than the median of both measures in the entire topic graph.

\section{CASE STUDY}

Our case study is focused on intermediary topics in the Chilean population on Twitter. In Chile, abortion is a sensitive issue, as the country has one of the strictest and severe abortion laws in the world. In the context of on-going campaigns for presidential elections, we crawled tweets from July 2013 to August 2013 using the Twitter Streaming API. ${ }^{1}$ Initially, we used query keywords about known issues and hashtags: abortion (issue), education (issue), gay marriage (issue), Michelle Bachelet (candidate), Evelyn Matthei (candidate), Santiago (location), among others. We also added emergent hashtags related to news events that happened during the crawling period. For instance, \#yoabortoel25 is about a protest held on July 25th.

In total, we crawled 4,611, 998 tweets from 768, 641 users. Of those tweets, 75, 432 from 40, 201 users were related to abortion. Of those users, only 7,518 reported a Chilean location in their profiles. Those users authored 1,962, 941 tweets about sensitive issues. We work with this subset of the crawled dataset.

Issues and Abortion Stances. We manually selected the top 200 keywords related to sensitive issues in Chile to define a corpus of documents built with the tweets containing their corresponding hashtags and keywords. Following our methodology, we built two stance vectors using relevant hashtags related to \#prochoice and \#prolife stances. Those vectors were weighted using TF-IDF according to the corpus. We estimated the user vectors for these users, by weighting the words used in their tweets according to the corpus. We estimated the user stances on abortion by computing the cosine similarity between user vectors and the stance vectors, displayed in aggregated form in Figure 1 left: \#prochoice similarity in $x$ and \#prolife similarity in $y$. We observe two kinds of users: polarized (those who employ vocabulary from one stance only, or few vocabulary from both) and nonpolarized (those who employ vocabulary from both stances and are situated on the upper-right part of the diagonal in Figure 1 left). It is surprising that many users lie on the diagonal of the plot, as showcased by the color intensity. Those findings are discussed in Section 4.

Intermediary Topics. In addition to our initial dataset, we crawled 2, 521, 113 tweets authored or retweeted by our user pool from December 6th, 2013 until January 3th, 2014. Then, we created user documents by concatenating those tweets and the tweets they have published before about sensitive issues. We ran LDA with $k=300$ and built the topic graph, with 234 nodes and 4,716 edges remaining after filtering. Betweenness centrality has a mean value of $0.0098,0.0199$ std. dev and 0.0041 median, and diversity has a mean value of 0.4855 with 0.0387 std. dev., and a median of 0.4861 . We estimated diversity considering a binary classification of user stances, based on the stance with the highest similarity for

\footnotetext{
${ }^{1}$ https://dev.twitter.com/docs/streaming-api. Data
} was crawled by the first author.
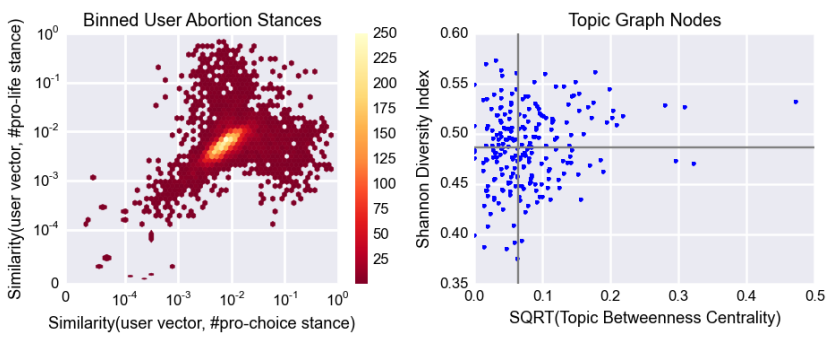

Figure 1: Left: Binned user stances: similarities between user vectors and stance vectors of \#prochoice in $x$ and \#prolife in $y$. Right: Centrality and Shannon entropy from the topic graph nodes. Grey lines depict the medians of each variable.

each user. Topics with centrality and diversity above the corresponding medians are what we define as intermediary topics. They are displayed in the upper-right quadrant of Figure 1 right. A manual inspection of words contributing to these topics reveals that most of them are about trending topics and non-sensitive conversations (music, places, etc.).

\section{DISCUSSION AND CONCLUSIONS}

Although Chile is a highly polarized country on a number of sensitive issues, in particular abortion, we found that user polarity is lower than expected, and that many users employ vocabulary related to both abortion stances. A possible explanation is that our TF-IDF weighting used in the stance vectors is softening the stronger stances on the issue. Nonetheless, intermediary topics do exist and are measurable. The existence of these topics is important as we can make use of them to enhance current social networks with mechanisms to connect users based on partial homophily while still providing a degree of diversity.

Future Work. The main question to target is for whom intermediary topics should be used. The distribution of user stances hints that intermediary topics, as defined here, can be useful to recommend content to polarized users. To confirm this, we will revisit the computation of the stance vectors to minimize artifacts and softening of stances. Next, we will define how to evaluate qualitatively the diversity of found intermediary topics, as popular terms might not be really diverse. Finally, evaluations of recommendations using intermediary topics will be challenging as user reception can be different than in a relevance-only context.

Acknowledgments. This work was partially funded by Grant TIN2009-14560-C03-01 of the Ministry of Science and Innovation of Spain.

\section{REFERENCES}

[1] David M Blei, Andrew Y Ng, and Michael I Jordan. Latent dirichlet allocation. the Journal of machine Learning research, 3:993-1022, 2003.

[2] Q Vera Liao and Wai-Tat Fu. Beyond the filter bubble: interactive effects of perceived threat and topic involvement on selective exposure to information. In Proceedings of the ACM CHI, pages 2359-2368, 2013.

[3] Sean A Munson and Paul Resnick. Presenting diverse political opinions: how and how much. In Proceedings of the ACM CHI, pages 1457-1466, 2010.

[4] Jianshu Weng, Ee-Peng Lim, Jing Jiang, and Qi He. Twitterrank: finding topic-sensitive influential twitterers. In Proceedings of the third ACM WSDM, pages 261-270, 2010. 